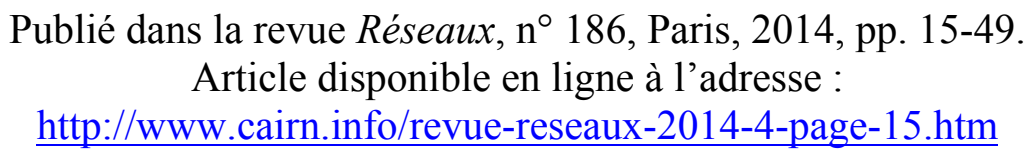

\title{
La déconnexion aux technologies de communication
}

Francis Jauréguiberry

En moins de vingt-cinq ans, nous sommes passés d'un plaisir récent de connexion à un désir latent de déconnexion. Lorsque les portables sont apparus, de même que les premiers ordinateurs personnels puis l'Internet, le mot d'ordre a été celui de la connexion. Présentées comme synonymes de progrès, d'ouverture, d'expériences créatives inédites et de nouvelles formes de participation citoyenne, les TIC sont alors tellement pleines de promesses que la non-connexion est très vite pensée comme une injustice : d'un côté ceux qui peuvent participer à la globalité de la société en réseau parce qu'ils ont accès aux équipements et connexions, et de l'autre les exclus qui n'ont pas cette possibilité. La notion de fracture numérique, telle qu'elle a été majoritairement exposée dans les années 1990, désigne cette inégalité et renvoie donc exclusivement à un problème d'accessibilité technique. Les inforiches sont ceux qui bénéficient de l'accès matériel aux réseaux et terminaux adéquats, et les info-pauvres sont ceux qui en sont privés.

$\mathrm{Au}$ fur et à mesure où, durant ma première décennie 2000, les TIC sont adoptées par un pourcentage grandissant de la population (que l'on pense par exemple au taux d'équipement en téléphones portables qui bondit en France de $4 \%$ en 1997 à $96 \%$ dix ans plus tard ou au pourcentage des internautes parmi la population de plus de 11 ans passant de $22 \%$ en 2001 à $69 \%$ en 2010), le thème de la lutte contre la fracture numérique semble s'estomper. Plusieurs chercheurs (par exemple Selwyn, Van Dijk, DiMaggio, Hargittai, Lenhart, Helsper ou Dutton) dépassent alors la première vision mécanique de la fracture numérique en proposant de prendre en compte les modes d'utilisation et les inégalités qui leur sont liées et qui renvoient non plus à une seule inégalité (avoir ou ne pas avoir accès aux TIC) mais à des inégalités multiples (savoir-faire et bénéfices). Il semble que ce soit Ester Hargittai qui, la première en 2002, ait appelé cette inégalité d'usage et d'appropriation le «second degré de la fracture numérique ». L'idée transversale est que la capacité des individus à s'approprier pleinement les TIC est très inégalement répartie et dépend grandement non seulement de leur capital économique, mais aussi de leur capital culturel et cognitif. Selon les travaux, ce sont plutôt le capital culturel et scolaire, le savoir-faire et les compétences techniques ou le milieu social et les réseaux de sociabilité qui sont mis en avant au moment de mesurer disparités discriminantes ${ }^{1}$.

\footnotetext{
${ }^{1}$ Périne Broccorne et Gérard Valenduc (2009) font une bonne synthèse de ces travaux en distinguant les compétences instrumentales (qui ont trait à la plus ou moins grande capacité à manipuler les matériels et logiciels), les compétences informationnelles (qui renvoient aux capacités à chercher, sélectionner, comprendre, évaluer et traiter l'information) et les compétences stratégiques (qui concernent l'attitude à utiliser l'information de manière proactive, à lui donner du sens dans son propre cadre de vie et à prendre des décisions en vue d'agir sur son environnent professionnel et personnel).
} 
Nous sommes donc ici loin de la seule problématique de l'accès qui a dominé les vingt dernières années du $\mathrm{XX}^{\mathrm{e}}$ siècle et qui, dans son élan majoritaire, renvoyait à deux présupposés. Techniciste d'abord : l'accès aux TIC devait conduire à leurs mêmes usages raisonnés. Et idéologique ensuite : plus il y aurait de communications (quantité) et plus il y aurait de communication (qualité). C'est cette confusion entre accès, usage et appropriation que ces études, presque toujours empiriques, font éclater. Ce n'est pas parce qu'il y a accès qu'il y a usages équivalents et ce n'est pas parce qu'il y a usages qu'il y a appropriations également bénéfiques. Selon les présupposés de la première fracture numérique, l'accès à la technologie devait conduire à gommer les inégalités. Exactement le contraire est démontré par cette nouvelle approche : non seulement l'accès aux TIC ne diminue pas les inégalités, mais, comme l'écrit Van Dijk (2005, p. 2) " plus [ces technologies] sont immergées dans la société et font partie du quotidien, et plus elles sont liées aux divisions sociales existantes ».

Mais la seconde fracture numérique elle-même ne parvient pas à rendre compte d'un autre phénomène qui, entre-temps, semble d'être emparé des « riches » de la première facture numérique: une sorte de nausée télécommunicationnelle et de fatigue technologique se traduisant par un désir de déconnexion. Lorsque celle-ci se donne à expérimenter, elle est rarement totale (abandon définitif de la technologie), la déconnexion est plutôt segmentée (dans certaines situations et à certaines heures) et partielle (seuls certains usages sont suspendus). Elle est portée par des personnes qui ne sont ni en difficulté économique (au contraire, elles appartiennent en majorité aux couches moyennes et supérieures), ni culturellement marginalisées (elles ont toutes un diplôme, un emploi et mènent plusieurs activités sportives ou culturelles), ni en déficit cognitif face aux technologies (elles les manipulent depuis des mois ou des années). Il est donc hors de question de les classer, selon une logique diffusionniste, parmi ce qu'Everett Rogers (1983) appellerait des « retardataires ». Pas plus que parmi ceux qui, vivant ruptures ou changement dans leur vie, en viennent à abandonner l'usage de ces technologies. Au contraire, il s'agit la plupart du temps de personnes parfaitement intégrées, très connectées et sachant parfaitement utiliser ces technologies ${ }^{2}$. Bien plus : ce sont souvent de gros usagers utilisant depuis longtemps ces technologies qui en viennent à adopter des conduites de déconnexion !

Les premières études (Jauréguiberry, 2003, 2005) menées sur ces pratiques de déconnexion ont permis de dépasser cette apparente contradiction en posant l'hypothèse de la surcharge informationnelle. C'est parce qu'il y a trop de branchements, trop de connexions, trop d'interpellations, trop de simultanéité, trop de bruits et trop d'informations qu'un désir de déconnexion apparaît. La déconnexion relève alors d'une volonté de ne pas se laisser aspirer par un tourbillon non maîtrisé d'informations et de communications. Cette hypothèse a largement été vérifiée et nous en rendrons compte, en particulier du fait que les formes de déconnexion partielles tendent toutes vers un idéal de la connexion maîtrisée. Mais, au fur et à mesure où les témoignages recueillis auprès de personnes se déconnectant de temps en temps s'accumulaient, est apparue une donnée que nous avions totalement sous-estimée. La déconnexion volontaire n'est pas seulement une sorte de fuite alimentée par un désir de souffler, de reprendre son rythme ou de préserver un temps à soi. Elle vise aussi, ne serait-ce que quelques heures, à se mettre à l'écart du monde, à prendre de la distance afin de " faire le point» et de «se retrouver ». Moment ou période de dialogue de soi à soi, de réflexivité, de confrontation avec le sens de sa vie, la déconnexion est alors vécue comme pratique de son intériorité et renvoie directement, nous en ferons l'hypothèse en fin d'article, à la notion de sujet dans une société hypermoderne.

\footnotetext{
${ }^{2}$ Il s'agit là de caractéristiques redondantes relevées dans le cadre de la recherche DEVOTIC (déconnexion volontaire aux technologies de l'information et de la communication), financée par l'Agence Nationale de la Recherche, coordonnée par l'auteur et qui a réunie pendant quatre ans une vingtaine de chercheurs: http://anr.devotic.univ-pau.fr. L'ensemble des verbatims de ce texte provient de terrains réalisés dans ce cadre.
} 


\section{1- L’impératif de connexion : deux logiques}

Deux logiques poussent constamment à la connexion aux $\mathrm{TIC}^{3}$. D'abord, celle de l'intégration et de l'appartenance. La conscience de la nécessité d'être intégré à un ensemble de réseaux de télécommunication pour exister socialement est une des données de base de nos sociétés hypermodernes. Certes, il est encore possible de s'en passer, mais de la même façon que les amish, mennonites ou autres technophobes se sont passés de certaines inventions durant le $\mathrm{XX}^{\mathrm{e}}$ siècle : en restant en dehors de ce qui (réseaux, moyens de transport physiques et télécommunications) permet l'innervation de nos sociétés et leur donne agilité et vitalité. La volonté de ne pas rester en dehors du social, de ne pas être exclu mais au contraire reconnu est à la base des motivations individuelles et non professionnelles à la connexion. Cet élan intégrateur, cette volonté « d'en être » et de ne pas «perdre le contact » n'est compréhensible que si l'on ne perd pas de vue le fantastique mouvement d'extension relationnelle et d'accélération des échanges qui s'est opéré durant la seconde moitié du XX siècle. Chacun veut profiter de «l'immense ouverture», synonyme de «choix identitaires » et «d'opportunités de rencontre » que notre société de communication met à sa portée. Chacun s'essaie donc à l'appartenance de ce qui lui paraît le plus profitable en fonction de ce qu'il perçoit de ses besoins, nécessités ou goûts du moment. Le cercle social d'appartenance, qui définissait assez bien l'individu jusqu'à peu, disparaît au profit d'un ensemble de bulles identitaires et occupationnelles plus ou moins éphémères et le plus souvent sans grande surface de recoupement entre elles. Il s'agit là d'un thème classique dans la sociologie actuelle : l'individu contemporain est pluriel, éclaté, polyvalent, voire kaléidoscopique ${ }^{4}$.

Dans la gestion de cette hétérogénéité identitaire et occupationnelle, les TIC arrivent sans doute à point nommé. Elles permettent de passer immédiatement d'une «tribu » à une autre, rendent plus certains et rentables les déplacements, multiplient les possibilités de rencontres et sont devenues indispensables pour coordonner les occupations au fil de l'eau. En activant le bon réseau au bon moment, il est possible de partager à distance et d'être reconnu malgré l'absence : l'intégration passe de plus en plus par la connexion. Continuer d'opposer comme aux débuts d'Internet une « réalité virtuelle » à la «vraie vie » revient non seulement à commettre un contresens (car la vie off line est tout aussi pleine de virtualité que la vie on line qui, elle, est tout aussi réelle que la vie off line), mais à passer totalement à côté de l'expérience contemporaine du monde qui ne cesse de mélanger les deux ${ }^{5}$.

Dans le même temps, les TIC servent aussi à resserrer des liens forts existant déjà malgré l'éloignement géographique ou le reflux des jours. J'avais montré dès le début des portables combien ceux-ci permettaient d'entretenir des liens forts contre ce qui dans notre quotidien éloignait et séparait ${ }^{6}$. Depuis, cette possibilité n'a cessé d'être approfondie, les appels où l'on n'a rien de précis à dire mais tout à communiquer se multipliant (au grand désespoir de ceux qui rabattent la communication au seul contenu des messages échangés). Le succès intégrateur des TIC est donc à chercher dans la possible synthèse qu'elles permettent entre ouverture et recentrage, zapping et continuité. Elles «chosifient» en effet en elles l'unité perdue des liens qui ne se recoupent plus, ni dans l'espace ni dans le temps. Objets

\footnotetext{
${ }^{3} \mathrm{Ne}$ sont ici traitées que les logiques alimentant les décisions individuelles à se connecter. Les logiques économiques et organisationnelles seront examinées plus loin. Les pressions commerciales, en particulier celles des opérateurs, des fabricants de matériels et des diffuseurs d'applications s'exercent aussi, évidemment, avec force.

${ }^{4}$ Voir par exemple, en français, Dubet (1994), Lahire (1998), Martuccelli (2010), Kaufmann (2004).

${ }^{5}$ Pour beaucoup, les choses les plus importantes se passant dans leur vie le sont désormais sur Internet. Internet n'est pas une réalité virtuelle mais fait partie de la réalité elle-même. Poser, comme le fait Sherry Turkle dans son ouvrage Alone Together (2011) qu'Internet éloigne de la "vraie vie » est selon nous un contre sens. La question est plus simplement de savoir quelle place chacun donne à Internet dans sa vie.

${ }^{6}$ Jauréguiberry, 1997.
} 
médiateurs, elles semblent condenser ce qui est épars et donc aider l'individu dans la gestion de son existence éclatée, de ses multiples appartenances, entre l'ici et l'ailleurs. Elles donnent aussi l'impression de ne pas se couper de ce que Georg Simmel appelait il y a déjà un siècle de cela les « multiples possibles » que la vie trépidante dans les grandes villes faisait entrevoir à l'homme moderne sans qu'il ne s'y engageât vraiment et dont il gardait ensuite la nostalgie ${ }^{7}$. Avec les smartphones, des contacts, numéros de téléphone, adresses e-mail sont stockés en attente de réactivations possibles. Nos amis sont sur Facebook, nos relations professionnelles sur Linkedin, les traces du passé sur Instragram et ceux que l'on a oubliés figurent sans doute sur les moteurs de recherche : tout n'est pas perdu, tout n'est pas lointain, tout est à portée de main.

La seconde logique poussant à la connexion est celle de l'efficacité et de la performance. Ici, ce n'est pas en termes de reliances affectives ou relationnelles que les motivations sont prioritairement exposées, mais d'efficacité, d'utilité, d'intensité, de gain et de rentabilité. L'ensemble des services proposés sur Internet en constituent un exemple patent et l'on voit mal comment nous pourrions désormais nous en passer. A-t-on bien mesuré combien e-mails, banques de données en ligne, centrales de réservation, webcams, applications dédiées et géolocalisées ont contribué à rendre notre vie plus aisée et notre quotidien plus pratique et performant en termes de budget temps/résultats obtenus ? La diffusion des smartphones permet d'avoir accès à ces services et données quel que soit l'endroit où l'on se trouve, les rendant encore plus efficaces dans la conduite de nos actions. Où se trouve la station de métro la plus proche ? Comment traduire cette indication en langue étrangère que je ne comprends pas ? Quel temps fera-t-il demain ? Reste-t-il des vélos en libre-service dans un rayon de 200 mètres ? L'information se trouve à portée de doigt, où que l'on soit, et la multiplication de capteurs électroniques dans notre environnement physique et de puces RFID collées aux objets rendra notre monde encore plus hybride si ce n'est bavard, nous suggérant des types d'utilisation, de parcours, de consommation ${ }^{8}$. En devenant informatifs sur la réalité environnante et en permettant d'avoir immédiatement accès aux banques de données mondiales, les smartphones se sont convertis en quintessence des technologies non seulement de communication mais aussi d'information et... d'action. Car ils servent aussi d'appareil photographique, de caméra, de lampe de poche, de réveil, de calculette, de jeux, etc. ${ }^{9}$

Si l'on ajoute à cela le caractère apaisant qu'une connexion peut apporter aux plus inquiets ( «en cas de problème ou de danger, j'appelle un spécialiste ou des secours »), on voit mal ce qui porterait à se déconnecter, les avantages semblant par avance l'emporter sur d'éventuels désagréments. Et il en va effectivement ainsi tant que l'on s'en tient à un niveau de libre choix individuel de se connecter. La connexion est une porte qui s'ouvre sur le monde et il paraît absurde de vouloir la refermer sur tant de possibilités, d'opportunités et de

\footnotetext{
${ }^{7}$ Simmel, 2013 [1903].

${ }^{8}$ Une question se pose alors : qui donne la parole à quoi et pour dire quoi ? Le pouvoir politique a-t-il encore quelque chose à dire sur l'hybridation informationnelle de biens publics à partir du moment où leurs usages informatifs deviennent privés ? Confiera-t-il cette tâche à des spécialistes, dont la logique n'est pas toujours celle des utilisateurs ou spécialement ouverte à la participation citoyenne ? Ou bien des services se mettront-ils en place de façon collaborative à partir des attentes et des expériences des usagers ? S'ouvrirait ainsi un énorme chantier d'écoute, de participations croisées et de mise en design de la réflexivité citadine. Si tel était le cas, des débats et pourquoi pas des luttes pour savoir qui décidera de quoi les objets et lieux doivent parler ont toute chance d'apparaître, la mise en place de nouvelles hybridités devenant ainsi un véritable enjeu social, politique et culturel.

${ }^{9}$ Posons ici une première distinction qui nous sera utile lorsque seront traitées les tactiques de déconnexion : l'on peut être déconnecté communicationnellement (téléphone et e-mails) et connecté informationnellement (presse, banque de données, services en lignes) ou l'inverse, ou encore être complètement déconnecté mais continuer à se servir de son smartphone comme réveil, dictaphone ou appareil photographique.
} 
promesses. Mais lorsqu'une partie de ce monde met le pied dans l'entrebâillement de cette même porte pour vous surveiller, vous ensevelir d'informations dont vous n'avez que faire ou encore pour vous vendre des choses que vous ne voulez pas, alors apparaît le désir de la refermer si ce n'est de la claquer.

\section{2- Les dangers d'une connexion non maîtrisée ou imposée}

En quelques années seulement et sans que nous n'y prenions vraiment garde, le fait de ne pas répondre immédiatement à son téléphone portable en est venu à devoir être justifié. Il faut s'expliquer, se dédouaner voire s'excuser de son absence de réactivité. Et si la déconnexion relève désormais de la justification, c'est bien que la connexion s'est entre temps convertie en norme. La normalité est de décrocher immédiatement son téléphone, surtout s'il est portable, la normalité est de répondre dans la demi-journée voire dans certains cas dans l'heure aux e-mails reçus et il y a de fortes chances pour que la géolocalisation de chacun devienne très vite, elle aussi, tout aussi normale.

Ce nouvel impératif à l'immédiateté télécommunicationnelle a d'abord été pris dans la sphère économique et dans l'activité professionnelle. Adaptabilité au marché, optimisation des fonctions et organisation du système de l'entreprise-réseau, à chacun de ces niveaux stratégiques les télécommunications jouent un rôle central et toutes les innovations dont elles ont été l'objet ces deux dernières décennies ont immédiatement été mobilisées dans un même but : raccourcissement des délais, accélération des rythmes et généralisation de la simultanéité. Dans la guerre économique que se livrent les acteurs de la chrono-compétitivité, elles ont été converties en de véritables armes. Mais cette accélération du temps n'est pas propre à la sphère économique. Elle touche tous les domaines de la vie, et en particulier la vie privée des individus. D'abord parce que le temps de travail donne encore largement, et quoiqu'on en dise, le rythme de la vie et qu'il est impossible d'y échapper. Ensuite parce que des catégories entières de salariés sont rattrapées par leur travail sur le terrain même de leur vie privée. La distance physique, les murs et les horaires de travail ne protègent plus : chaque jour davantage, l'urgence professionnelle fait irruption dans l'espace privé en le « trouant » sous la forme d'astreintes, de gardes ou de parenthèses téléphoniques ${ }^{10}$. Enfin, la contagion de l'urgence à l'espace privé relève aussi d'un phénomène sans doute moins visible, mais pas moins profond : la déteinte du mode de fonctionnement professionnel sur le mode d'existence privée. De façon diffuse, les modes d'action repérables dans la sphère économique (pragmatisme, utilitarisme, compétition, rentabilité, efficacité, désir de gains et de puissance) sont appliqués à ce qui est en passe de devenir la « gestion » des occupations et relations privées : la vie est devenue un capital qu'il s'agit de faire fructifier au plus vite ${ }^{11}$.

La chasse au temps perdu, le culte du potentiel, la montée de l'urgence, la pression de l'immédiat et la multiplication des informations plongent l'individu dans une situation d'injonctions répétées à réagir de plus en plus vite. Cette accélération peut être vécue de façon positive : comme multiplicateur d'activités et d'opportunités, comme révélateur de certaines potentialités organisationnelles jusqu'alors inexploitées, comme agent de simplification ou encore comme réhabilitation de l'intuition individuelle dans la gestion des affaires. Elle peut aussi être source de satisfactions, en particulier pour certains professionnels indépendants qui, vivant cette course au temps sous la forme de défis renouvelés, la trouvent passionnante et parfois gratifiante. Mais cette accélération peut aussi donner le vertige, et la chute n'est alors pas exclue. Dans un monde où tout s'accélère et se bouscule, l'individu placé dans cet état de tension et d'urgence quasi permanentes court en effet deux risques.

\footnotetext{
${ }^{10}$ L'inverse est aussi vrai : appels téléphoniques, e-mails et SMS traités pendant le temps de travail se sont multipliés.

${ }^{11}$ Ce paragraphe reprend un développement déjà exposé dans Jauréguiberry (2005).
} 
Le premier de ces risques est de réagir à l'impulsion afin d'éviter ce qu'on peut appeler "l'effet bouchon»: l'accumulation incontrôlable d'informations interdisant leur traitement efficace. Face à une pile de notes, à une succession d'e-mails urgents et à une multiplication des sollicitations téléphoniques, il faut aller vite. Non seulement il faut toujours être connecté, mais il faut aussi pouvoir répondre rapidement. Dans cette accélération, synonyme de diminution du temps de réflexion, l'accessoire risque de recouvrir l'essentiel. Outre le stress lié à l'activité fébrile qui en résulte, le danger d'une telle réaction est de voir remplacer la réflexion et l'imaginaire par une espèce de réflexe à parer au plus pressé. L'homme pressé se convertit en pompier cherchant à éteindre le feu de l'urgence là où il prend. Le coup de fil a dès lors priorité sur la personne présente, le courrier électronique sur le dossier en train d'être traité, et le bip d'un SMS arrête tout, séance tenante. Comme si l'individu se mettait aux ordres de l'urgence, l'advenant supplantant l'existant.

Il est évidemment des situations dans lesquelles nécessité fait loi. Mais l'extension de ce mode de réaction menace de le transformer en une véritable mode de fonctionnement avec les dangers que cela entraîne. Focalisées sur la réaction aux sollicitations immédiates, personnes et structures courent en effet le risque de perdre tout pouvoir stratégique au profit de pures tactiques d'adaptation à un environnement qu'elles ne maîtrisent plus. Dans ce cas, l'information devient bruit, la vitesse précipitation, et les passages à l'acte font office de décisions. Ce type de conduite risque de rendre obsolète la notion même de projet. Le projet nécessite une certaine confiance dans l'avenir. Il relève d'un " pari » sur le futur en pensant que l'action programmée pourra s'y déployer. Il n'y a évidemment, en la matière, aucune certitude : seule la confiance permet de différer, de planifier, de se représenter ce qui sera contre ce qui est. Mais si le présent lui-même apparaît comme indéterminé, n'est plus vécu que comme immédiateté éphémère, comment penser le projet dans sa durée ? Dans un monde où il faut s'adapter rapidement, comment peut-on encore adapter le monde dans la durée ?

Le second risque est de se mettre à hésiter dans l'urgence. Les prises de décision deviennent dans ces conditions autant de violences que l'individu s'impose dans une situation qu'il ne maîtrise plus. Tensions, stress et parfois même anxiété ont alors toutes chances d'apparaître. L'individu sous tension se sent non plus interpellé mais harcelé. Tout un ensemble de pathologies psychosomatiques désormais parfaitement connues sont associées à cette contradiction dans laquelle se place l'individu en « surchauffe » occupationnelle. D'un côté la conscience de l'urgence, de l'autre celle de ne pouvoir y faire face, ce qui ne fait qu'augmenter la pression qui s'exerce sur lui. Il est même des cas où l'individu reste comme groggy devant trop d'interpellations. Pour lui, la seule façon de ne pas cesser d'exister complètement est alors de «craquer». Entrant dans un véritable état de catalepsie, il démissionne par overdose communicationnelle. Burn out, vide apathique ou dépression, il s'agit toujours de conduites de repli quand la pression devient trop forte, que les charges se révèlent insupportables et que les moyens pour y faire face paraissent brusquement dérisoires. À l'issue de telles situations de stress qui l'ont épuisé, usé, érodé, l'individu démissionne ${ }^{12}$.

Toute gestion en temps réel ou tout pilotage en situation d'urgence ne saurait évidemment conduire à une telle issue dramatique. Non seulement parce que les réactions à l'urgence peuvent avoir été planifiées de façon préalable (mise en place de procédures, fruits d'anticipations stratégiques), mais aussi, de façon plus fondamentale, parce que ces réactions peuvent relever d'une rationalité, certes limitée par le temps, mais non hétérogène à une continuité stratégique pourvoyeuse de sens. Mais l'extension de ce mode de réaction à des aspects de plus en plus divers de la vie quotidienne menace de le transformer en un véritable mode de fonctionnement. Focalisé sur la réaction aux sollicitations immédiates dont il est l'objet, l'homme pressé court dans ce cas le risque de souffrir de ce que j'ai appelé le

\footnotetext{
${ }^{12}$ Sur ce thème, voir Nicole Aubert (2003).
} 
« syndrome du zappeur ». Par syndrome du zappeur, j'entends l'ensemble des symptômes du mal latent qui guette ceux qui vivent leur expérience d'instantanéité médiatique selon une logique de pure rentabilité au point de s'y faire absorber. C'est tout à la fois l'anxiété du temps perdu, le stress du dernier moment, le désir jamais assouvi d'être ici et ailleurs en même temps, la peur de rater quelque chose d'important, l'insatisfaction des choix hâtifs, la hantise de ne pas être branché au bon moment sur le bon réseau, et la confusion due à une surinformation éphémère.

Le syndrome du zappeur, c'est le mal des télécommunications dont est pris l'individu hyperconnecté lorsqu'il tombe dans ce que plusieurs de nos informateurs ont appelé le «tourbillon». Comme soumis à l'effet d'une force centrifuge due à une accélération non maîtrisée, l'individu est dépossédé du sens de son action et aspiré par une inflation occupationnelle. Contraint à réagir sur le mode de l'urgence à une masse d'information grandissante et à des aléas de plus en plus fréquents, il se trouve réduit à s'accrocher où il peut s'il ne veut pas sombrer, à faire des coups et à développer des tactiques de nature opportuniste. L'acteur est alors moins mû par une logique de type stratégique, visant à adopter les meilleurs moyens par rapport à des fins qu'il s'est fixées, que par une logique de type tactique, visant à s'adapter au mieux à une situation qu'il ne domine plus.

C'est à partir de ce constat fait lors de précédents terrains et de l'observation du malaise, voire dans certains cas de la souffrance que cela engendrait, qu'avait été posée, en amont de la recherche DEVOTIC, l'hypothèse que les conduites de déconnexion étaient principalement motivées par une volonté d'échapper à ce tourbillon.

\section{3- La déconnexion comme fuite}

Les cas de déconnexion les plus spectaculaires, ceux dont la presse s'est faite écho et qui ont donné lieu aux témoignages les plus diffusés se placent majoritairement sur cette première piste. Ainsi, les expériences de déconnexion de Thierry Crouzet (J'ai débranché, 2011) ou de Susan Maushart (The Winter of our Disconnect, 2010) qui ont été largement commentées, relèvent d'une tentative d'échapper à une overdose de connexion. Le premier parle de « désintoxication » et de « sevrage », la seconde de " ras le bol » et de « désir de faire une pause $»{ }^{13}$ Il s'agit de conduites de fuite, de crise, de ras-le-bol dans des situations de sursollicitations. Le désir de déconnexion apparaît dans des situations de saturation, de trop-plein informationnel, de débordement cognitif, de harcèlement ou de surveillance dans lequel l'individu se sent dépassé ou soumis. La pression transitant par les TIC, il y a comme un espoir diffus qu'en bridant celles-ci, celle-là s'en trouvera diminuée. Dans les cas extrêmes de burn out, le rejet des TIC fait partie intégrante d'attitudes de défenses ultimes qui permettent à l'individu de survivre quand il ne peut plus lutter. Ces cas sont heureusement rares et relèvent moins d'une déconnexion volontaire cherchant à maîtriser des flux communicationnels que d'une déconnexion mécanique visant à ne pas se laisser emporter par un mouvement incontrôlable. À l'image d'un disjoncteur qui saute lorsque l'intensité électrique devient trop importante, la déconnexion est ici purement réactive. Les témoignages des personnes ayant traversé un épisode de burn out montrent tous que la déconnexion n'est pas volontaire mais automatique, au même titre que l'arrêt immédiat du travail.

\footnotetext{
${ }^{13}$ Pendant trois ans, 312 articles de presse et blogs portant sur la déconnexion ont été répertoriés sur le site DEVOTIC (si l'on compte les commentaires postés sur les blogs, on arrive à un corpus d'environ 500 témoignages). L'analyse sémantique à laquelle s'est livrée Nicole Lompré sur ces articles et blogs montre que, dans l'immense majorité, la déconnexion est corrélée au thème de l'hyper-connexion et de la surcharge informationnelle (une petite partie pose la déconnexion comme forme de résistance à la surveillance et au traçage).
} 
Les burn out sont souvent présentés comme des formes d'effondrement de soi sous le poids de surcharges de travail ou sous la pression de sur-sollicitations. Mais les cas cliniques montrent qu'ils relèvent surtout de l'accumulation du sentiment de ne pas pouvoir y faire face ou de l'absence de raisons d'y faire face. «Ne pas pouvoir y faire face » signifie manquer de temps, de ressources cognitives, de capital culturel, d'énergie pour traiter un nombre d'emails ou de SMS manifestement trop grand pour être raisonnablement gérés, ou pour répondre à un nombre d'appels téléphoniques trop fréquents pour ne pas être perturbants, ou encore pour entretenir de façon satisfaisante ses réseaux sociaux sur Internet, c'est à dire sans avoir constamment l'impression d'être en retard. "L'absence de raison de le faire » signifie qu'à force d'être sollicité pour des choses urgentes et importantes devant être traitées toute affaire cessante mais se révélant ensuite insignifiantes, à force de subir l'injonction paradoxale de s'investir pleinement et durablement dans des projets dans un monde dominé par la flexibilité et l'adaptation, les raisons de croire s'érodent et celles d'espérer disparaissent. C'est dans cet état dépressionnaire que les burn out apparaissent. Les TIC ne les provoquent pas mais leur usage inconsidéré, c'est-à-dire constant, à flux tendu et sans aucune prise de recul, oui.

\section{4- La déconnexion volontaire}

Les conduites de déconnexion volontaires que nous avons observées se situent toutes en deçà de telles réactions extrêmes. Elles visent précisément à éviter de rentrer dans la zone rouge du burn out et de subir des situations de surcharge informationnelle insupportables. Les déconnexions volontaires apparaissent à partir du moment où le désir de déconnexion va audelà d'une plainte ou d'une fatigue («Je suis débordé », "Je n'en peux plus », "Je croule sous les e-mails ») et se traduit par des actions, des conduites et des tactiques effectives. Il s'agit par exemple de mettre sur off son téléphone portable dans certaines circonstances ou plages horaires, de déconnecter son logiciel de courrier électronique en choisissant de ne l'interroger que de façon sporadique, d'accepter de ne pas être constamment branché sur ses réseaux sociaux ou de refuser d'être géolocalisable où que l'on soit. Dans tous les cas, la décision apparaît après une prise de conscience, soit par accumulation (la situation n'est plus tenable, il faut faire quelque chose), soit par un incident critique (un événement qui décale d'un coup son point de vue $)^{14}$.

« Je suis obligé de traiter [les e-mails] au fil de l'eau (Claude, responsable des achats, magasin de meubles, 34 ans). J'en reçois trop [environ 80 par jour]. Je me suis rendu compte que ce n'était matériellement plus possible, parce que je ne faisais plus que ça (...). J'ai été plus vigilant et je me suis rendu compte qu'aucun ne pouvait pas attendre 2 heures, aucun! Alors je me suis donné une nouvelle règle : je ne relève que toutes les heures, $j$ 'essaie toutes les deux heures... c'est plus difficile, mais j'essaie (...). Ça change tout, parce que pendant une heure, une heure et demie, je n'ai plus cette distraction. Et les traiter à la suite, ça va plus vite. Il n'y a qu'une interruption. Avant, il y en avait dix par heure. »

« J'ai décroché de Facebook en même temps que j'ai arrêté l'iPad au lit... J'y étais tous les soirs, c'était devenu un rituel, une heure, parfois plus (...). [160 amis] ça fait beaucoup de choses à lire avec les liens, etc. Il y avait toujours une petite voix : « tu n'as rien de mieux à faire ?» Ben non, j'avais rien de mieux à faire. Mais quand même, ça représentait beaucoup de temps pour pas grand-chose (...). Je regardais ça comme la télé. Un soir, je me suis endormie avec mon iPad ouvert (...). J'ai arrêté du jour au lendemain, je n'étais pas contente de moi. » (Liliane, 50 ans, technicienne).

« J'avais instauré une méthode qui consistait à tout traiter au fil de l'eau. Ça me permettait de supprimer à peu près les trois quarts des mails sans les lire. Les autres, je mettais sur « répondre » et je les traitais

\footnotetext{
${ }^{14}$ Cindy Felio (2013) a consacré une partie de sa thèse (menée dans le cadre du projet DEVOTIC et portant sur les Pratiques communicationnelles des cadres. Usage intensif des TIC et enjeux psychosociaux) à l'étude de ces « incidents critiques » chez des cadres ayant décidé de se déconnecter, au moins partiellement. Il est remarquable que, pour certains d'entre eux, leur interview sur ce thème ait précisément constitué cet élément déclencheur...
} 
après. Mais je me suis aperçu que dans «brouillon », j'avais du coup des dizaines de mails en instance et que j'avais une sérieuse tendance à remettre certaines réponses au lendemain et à accumuler (...). Le problème était déplacé, mais était toujours là. Et le problème c'est qu'il y a trop de sollicitations et pas assez de temps pour y répondre ou pas assez d'énergie. » (Régis, universitaire, 58 ans)

La déconnexion n'est jamais définitive mais toujours ponctuelle, partielle et située dans des contextes où " trop » est trop, où « encore » ne fait plus sens et où «plus » devient insupportable... Il ne s'agit pas de renoncer aux TIC mais d'essayer d'en maîtriser l'usage en instaurant des coupures, des sas temporels, des mises à distance. Les déconnexions ne sont pas spectaculaires comme dans les cas de burn out, mais modestes, font partie du quotidien et sont parfois pratiquées avec attention. Une forme de déconnexion, très souvent évoquée, consiste à mettre son téléphone portable sur silencieux (et, soulignent certains, de le laisser dans son sac de façon à ne pas voir l'écran s'allumer lorsqu'il y a un appel entrant), une autre à laisser son ordinateur portable au bureau. Sortir pour faire ses courses, prendre un café ou faire son jogging sans portable sont autant de façons de se déconnecter, tout comme décider de ne pas relever ses e-mails le temps d'un week-end. Il s'agit là de " petites déconnexions ", non spectaculaires et dont on ne parle pas dans les médias mais qui constituent l'essentiel pour ne pas dire la quasi-totalité des déconnexions.

La lecture des retranscriptions des dizaines d'heures d'entretiens menés auprès de ceux qui se déconnectent permet de dégager toute l'importance que ceux-ci accordent au thème du temps dans leur motivation à se déconnecter. Les TIC sont de fantastiques outils de gestion du temps. Elles permettent en effet de mieux coordonner nos actions, rendent nos emplois du temps beaucoup plus agiles et précis. Le fait de pouvoir être immédiatement informé d'aléas de dernière minute, des horaires des transports en commun, de rendez-vous déplacés, etc. est synonyme de gain de temps, d'économie d'énergie et souvent soldeur de stress. Pour s'en tenir au smartphone, il devient un agenda intelligent : taper une heure ou une date dans un email y renvoie automatiquement, évitant ainsi oublis ou recherches fastidieuses. Pour peu que l'on soit sur un Cloud, l'information circule sur l'ensemble des terminaux que l'on utilise. Au total, les TIC devraient donc permettre de gagner du temps. Pourtant, c'est exactement de l'inverse dont se plaignent ceux qui se déconnectent !

Car, en ne cessant de "pousser» vers eux des informations et sollicitations non attendues, les TIC obligent leurs utilisateurs à les traiter. Cette obligation peut être imposée : par sa hiérarchie, son conjoint ou ses amis. L'obligation est professionnelle, statutaire ou relationnelle. Les TIC ne créent pas ces liens de dépendance mais les densifient au point de les rendre parfois trop lourds à porter. Ainsi les e-mails et SMS trop pressants d'un supérieur ou d'un collègue, les appels trop fréquents d'un conjoint ou d'une mère inquiète, ou encore les notifications trop nombreuses de ses réseaux sociaux produisent à la longue une usure, une fatigue, une érosion qui se traduisent par des moments d'exaspération, des sautes d'humeur, un sentiment de trop-plein ou de débordement. C'est dans ce cadre-là qu'un temps déconnecté en vient à être pensé. Un «temps à soi » dans lequel l'individu peut retrouver ses propres rythmes, le sens de la durée et de l'attente, de la réflexion et de l'attention.

\section{5- Déconnexions professionnelles}

Professionnellement, il y a des emplois pour lesquels la déconnexion n'est tout simplement pas possible pendant les heures de travail. Tous les emplois liés à la veille commerciale (appels de clients potentiels), aux renseignements et réservations, ou à des fonctions d'intervention rapide répondent par exemple de cette obligation. Mais les TIC ont en l'espace de quelques années été le vecteur par lequel une surcharge informationnelle s'est ajoutée à des emplois qui, normalement, demandent une certaine concentration, nécessitent une continuité d'exécution et n'impliquent en rien une connexion continue. La mise en demeure de rendre compte quasiment au fil de l'eau de la progression de son travail, les 
contrôles multiples en cours même d'exécutions des tâches et le culte de l'information tous azimuts dans l'entreprise perturbent et souvent déstabilisent au point qu'un nouvel enjeu est apparu : le droit à la déconnexion.

Ce droit, ou plutôt sa revendication, s'est dégagé comme réaction dès que les technologies de communication ont ouvert de nouvelles « zones d'incertitude» au sein des entreprises ${ }^{15}$. Ces zones ont en effet immédiatement été investies par les directions dans le but de rendre le travail des employés encore plus productif, de pouvoir en contrôler l'effectivité et d'en augmenter l'intensité. Les premières revendications sont toutes apparues devant trop de contrôles, trop de transparence imposée et trop de sollicitations. Contrairement à ce que pourrait laisser penser une vision panoptique de l'organisation, des zones d'opacité sont nécessaires à son bon fonctionnement, car elles constituent la base même de la responsabilisation des employés. Ainsi, et pour ne prendre qu'un seul exemple sur lequel Luc Bonneville et Sylvie Grosjean (2012) ont travaillé, les infirmières de deux établissements de santé de Montréal ont débrayé à plusieurs reprises afin de faire annuler une décision qui leur imposait de rendre constamment compte de leur tâche par le biais de tablettes qui les suivaient partout. Du point de vue de la rationalisation des services et du suivi des soins, cette nouvelle disposition avait un sens théorique. Mais de façon pratique, les infirmières se sentaient non seulement contrôlées à distance, mais constamment menacées de devoir quitter leurs patients pour effectuer une autre tâche jugée plus urgente selon une vision panoptique du service. La nouvelle télé-organisation rendait ainsi superflues les micro-périodes d'attente entre deux patients et de recouvrement entre deux gardes. Ces périodes étaient pourtant vécues comme essentielles du point de vue des infirmières : remplissage de fiche de suivi des patients, mais aussi moments de rencontre avec les autres infirmières. Ces moments leur permettaient de partager des savoir-faire et d'échanger des informations qu'elles considéraient comme inhérentes à l'exercice de leur métier, mais aussi de pouvoir moralement s'épauler en cas de difficultés souvent rencontrées. À partir du moment où tout était (sensé être) dit par voie électronique, ces échanges n'étaient officiellement plus nécessaires et donc assimilés à une perte de temps. On voit comment l'idéal de la transparence et du zéro temps mort, qui semble accessible grâce à ces technologies permettant la connexion permanente et la simultanéité, se heurte à la réalité d'un métier qui nécessite des zones d'autonomie pour pouvoir exister. Et c'est pour retrouver ces zones d'autonomie que certains n'hésitent pas à se déconnecter.

«A force de répondre à tous ces mails, j'avais perdu mon métier, j'avais perdu le sens de ce que je faisais. Je répondais à des mails qui n'avaient rien à voir avec ce que je faisais (...). Et tous les jours, je passais de plus en plus de temps à répondre en pensant que ça ne servait à rien (...) Le pire, c'est que j'avais l'impression que je faisais quelque chose, mais que ce quelque chose me volait mon temps, mon vrai temps de travail (...) ça ne pouvait pas durer comme ça. » (Claire, secrétaire, 41 ans)

«Le fait même de me déconnecter m’a amené à mieux hiérarchiser. Plusieurs fois, je me suis rendu compte que si [je ne m'étais pas déconnecté], je n'aurais pas décidé de faire telle ou telle chose en premier. En me reconnectant, c'est elle que je faisais en premier. Elle était devenue une priorité parce que j'y avais réfléchi entre temps. Sinon, elle aurait été perdue au milieu des autres toutes liées à la liste des e-mails. Et dans la liste des e-mails, on ne traite pas en priorité ce qui est le plus important, ça ne marche pas comme ça, on se laisse aller... » (Jean, 42 ans, chargé d'étude dans un cabinet-conseil)

«Quand je suis sur un dossier (35 ans, notaire dans une étude d'une cinquantaine d'employés), je ne peux pas faire trente-six autres choses. Mais je répondais [au téléphone] et après il me fallait toujours

\footnotetext{
${ }^{15}$ Par «zones d'incertitudes » Michel Crozier entend des situations où les besoins mêmes des actions qui s'y déployant créent de l'imprévisibilité. En particulier, Crozier (1971) montre que les relations de pouvoir au travail sont constamment l'objet de négociations, directes ou détournées, où chacun cherche à accroître la marge d'imprévisibilité de son comportement vis-à-vis de son adversaire. En effet, plus la zone d'incertitude de l'action d'un employé est grande vis-à-vis de sa hiérarchie, et plus il a de pouvoir (pouvoir d'initiative et d'agir comme il l'entend sans crainte de sanction). À l'inverse, plus la hiérarchie est capable de réduire l'imprévisibilité du comportement de l'employé, et moins il aura de pouvoir.
} 
cinq minutes pour m'y remettre, à chaque fois... sans compter les appels qui m'obligeaient à faire autre chose entre temps. Depuis un an (le fait d'avoir changé de bureau a peut-être joué), je laisse presque toujours sonner... Je vous emmerde, je travaille... Deux fois sur trois, il n'y a pas de message [sur le répondeur] et pour le reste, ce n'est jamais si urgent, je rappelle (...) J'ai retrouvé une sorte de continuité dans mes dossiers, je vais plus vite et je suis plus concentré. »

« Je ne suis ni la concierge, ni la standardiste, ni le DRH. Mais [parce que j'ai un e-mail et un portable], je dois lire des mails qui ne me concernent pas, répondre à des appels qui n'ont rien d'urgent, régler des problèmes d'occupation de salle, de rendez-vous reportés... Depuis [que j'ai instauré mes heures de déconnexion $]^{16}$, j'ai retrouvé la sensation de réellement faire quelque chose. Au bout de mes deux heures, je me dis : j'ai fait ça, ça et ça. Je sais ce que j'ai fait. » (44 ans, chef d'un service de trente employés dans une institution de plus de 800 personnes)

On le voit, chacun improvise comme il peut des usages professionnels soutenables des TIC impliquant des moments de déconnexion... Il semble que les dangers d'une mise en télédisponibilité permanente, rendant en particulier la distinction entre vie professionnelle et vie privée de plus en plus poreuse, aient vite été signalés. Par exemple, la Communauté européenne, dans sa «recommandation 6c » sur la société de l'information (1997, p. 51) signalait dès la fin des années 1990 qu'il convenait d'être attentif et d'étudier «les conséquences d'une connexion permanente, le besoin de se déconnecter parfois et le droit de restreindre l'accès à certains moments » tout comme «la restriction et la négociation des intrusions ». De même, la Cour de cassation a depuis rendu en France plusieurs arrêtés allant clairement dans le sens d'un droit à la déconnexion ${ }^{17}$. Celui-ci est une des propositions centrales de la CFDT Cadres: «Des négociations doivent s'engager avec les organisations syndicales, dans les entreprises et les administrations, pour prendre en compte les nouvelles réalités professionnelles (introduites par les technologies de la communication) et faire reconnaître pour chaque salarié un véritable droit à la déconnexion, en particulier pour éviter les astreintes insidieuses ». De timides expériences commencent à aller dans ce sens, par exemple Volkswagen qui en Allemagne interdit tout e-mail professionnel après $18 \mathrm{~h}$ et les week-ends ou, tout dernièrement en France, l'accord de branche concernant les sociétés d'ingénierie et de conseil spécifiant une " obligation de déconnexion ». Il semble qu'après une vingtaine d'années d'idéologisation des technologies de la communication (les assimilant à quelque chose d'intrinsèquement bien, le mal étant en la matière le silence, l'écran noir et la déconnexion), d'injonction à «communiquer plus» et de pression à être constamment connecté, soit venu le temps d'une réflexion sur ce qui parait soutenable en termes de capacités psychologiques et souhaitable d'un point de vue social et organisationnel ${ }^{18}$.

C'est au demeurant davantage ce second point (danger de contre-performance) que le premier (risques psychosociaux) qui a motivé les premières études managériales sur la déconnexion. Les cas de dysfonctionnement commençant à très sérieusement inquiéter quant à leur coût, les directions en vinrent à se poser la question de déconnexion pour des raisons de

\footnotetext{
${ }^{16} \mathrm{~S}$ 'est imposé (et a eu le pouvoir d'imposer aux autres) deux heures de déconnexion (courrier électronique et téléphone) le matin.

${ }^{17}$ Par exemple, celui du 10 juillet 2002 spécifiant que le temps de repos «suppose que le salarié est totalement dispensé directement ou indirectement, sauf cas exceptionnels, d'accomplir pour son employeur une prestation de travail même si elle n'est qu'éventuelle ou occasionnelle ", ou encore celui du 17 février 2004 où il est clairement indiqué que « le fait de n'avoir pas pu être joint en dehors des horaires de travail sur son téléphone portable est dépourvu de caractère fautif et ne permet donc pas de justifier un licenciement disciplinaire pour faute grave ».

${ }^{18}$ Dans sa thèse (Techniques de l'Information et de la Communication et risques psychosociaux sur le poste de travail tertiaire, menée dans le cadre du projet DEVOTIC), Thierry Venin (2013) a parfaitement montré les dégâts de cette idéologie appliquée à l'organisation des postes de travail dans le tertiaire : des catégories entières d'employés, pris dans une espèce de folie technicienne passant leur temps à adapter leur travail à des matériels et aux dernières versions de logiciels très vite obsolètes.
} 
stricte performance managériale ${ }^{19}$. Un sondage mené en 2003 auprès des dirigeants d'entreprises en France montre qu'ils ont été très vite conscients des effets potentiellement négatifs (en termes de manque de vision et de réflexion stratégique) des technologies de la communication ainsi que du sentiment d'urgence permanente qu'elles pouvaient susciter ${ }^{20}$. Leurs préoccupations rejoignent en cela les conclusions des études menées en sciences de la gestion autour de la notion de surcharge informationnelle (information overload) ${ }^{21}$. Mais l'essentiel de ces études est mobilisé dans le cadre général des théories de la décision et selon une vision utilitariste: jusqu'à quel point peut-on informationnellement charger ses collaborateurs sans que cela n'engendre des effets négatifs ? La surcharge informationnelle et cognitive est envisagée comme ayant un impact sur la rationalité des décisions organisationnelles et la stratégie globale des entreprises dont il s'agit de mesure les aspects négatifs $^{22}$. Certains ont bien saisi l'opportunité qui s'ouvrait de prévenir les dangers sous forme de conseils : ouvrages ${ }^{23}$, stages de déconnexion, coaching es-déconnexion et autres applications de déconnexion font florès ${ }^{24}$. Mais peu d'attention est consacrée aux questions du lien organisationnel et aux métamorphoses des modes de communication, pourtant support de l'engagement ou du désengagement des salariés, ni au stress que ces pratiques peuvent induire.

Les pratiques de déconnexion demeurent donc, dans l'écrasante majorité des cas, individuelles. Or tous les employés et cadres ne sont pas égaux dans leur latitude et leur capacité à mettre ces pratiques en œuvre. Si l'on voulait forcer le trait, on pourrait écrire qu'il y a d'un côté ceux qui ont le pouvoir de se déconnecter, et donc d'imposer aux autres leur (relative si répondeur) inaccessibilité, et de l'autre ceux qui ne l'ont pas ; d'un côté ceux qui ont le pouvoir d'imposer aux autres une disponibilité d'écoute permanente, et de l'autre ceux qui doivent se plier à cette volonté ; d'un côté donc ceux qui ont le pouvoir de se déconnecter et de l'autre ceux qui ont le devoir de rester connecté. Comme nous le notions déjà en $2003^{25}$ : les nouveaux pauvres des télécommunications ne sont plus ceux qui n'ont pas accès à la connexion, mais ceux qui vivent désormais dans l'obligation de répondre immédiatement et qui ne peuvent donc pas échapper à la situation de vivre dans une sorte d'interpellation continue. Tandis que les nouveaux riches des télécommunications sont ceux qui ont la possibilité de filtrer et donc d'instaurer de la distance vis-à-vis de cette même interpellation.

Bien entendu, ce ne sont pas les TIC qui créent de toutes pièces cette inégalité. Celle-ci dérive de la hiérarchie, des rapports de force, des statuts, et en définitive des types de pouvoir déjà existants au sein des entreprises, organisations ou réseaux. La question n'est donc pas de considérer les technologies de communication comme producteurs sui generis de nouvelles

\footnotetext{
${ }^{19}$ En anglais cela donne des titres évocateurs. Par exemple « Digital Overload: Too Much Technology Takes a Toll» (Business News, 8 novembre 2010) ou « Digital Overload: Is Your Computer frying Your Brain?» (Livescience, 9 novembre 2010).

${ }^{20}$ Réalisé pour Microsoft par Adecco. $81 \%$ pensent que « les technologies de communication ont créé un nouveau sentiment d'urgence, lié à la rapidité de transmission de l'information et aux attentes qui en découlent » et $73 \%$ pensent que cet état provoque «un manque de recul pouvant entraîner des décisions non pertinentes prises dans le mouvement ».

${ }^{21}$ Martin J. Eppler et Jeanne Mengis (2004).

${ }^{22}$ Henri Issac, Michel Kalika, Eric Campoy (2007).

${ }^{23}$ Par exemple le best-seller de Joanne Cantor Conquer CyberOverlooad (2010) décrivant tout un ensemble de recettes pour éviter le burn out.

${ }^{24}$ Par exemple Ommwriter conçue par Rafa Soto et Marzban Cooper et au départ conçue « pour solutionner un problème interne que l'on avait dans notre entreprise : la difficulté de se concentrer lorsqu'il s'agissait d'écrire sur son ordinateur. Avec les notifications d'e-mails, SMS, Twitter, Face-Book, les distractions étaient en effet continuelles et émoussaient notre lucidité et attention sans que l'on s'en aperçoive. " (El Pais, 21 avril 2011, p. 50). L'application qu'ils mettent alors au point et qui consiste à stocker les « distractions » sans possibilité d'y avoir immédiatement accès, a été téléchargée plusieurs centaines de milliers de fois en quelques mois...

${ }^{25}$ Jauréguiberry (2003), p. 104.
} 
formes d'exploitation, mais de savoir si la simultanéité télécommunicationnelle qu'elles autorisent tend plutôt à renforcer les inégalités existantes (sous la forme du couple contrôle/dépendance) ou au contraire à les émousser (en permettant d'expérimenter de nouvelles formes d'organisation dans le sens d'une plus grande autonomie et responsabilité de chacun).

\title{
5- Déconnexions privées
}

C'est le point des déconnexions privées que les terrains menés pour le projet DEVOTIC nous ont le plus surpris. Nous ne pensions pas, qu'enquêtant sur des pratiques de déconnexion, nous recueillerions des considérations aussi graves et profondes. La déconnexion ne renvoie rien moins qu'au sens de la vie, aux questions existentielles et à la force des engagements. Nous ne nous attendions pas du tout à l'irruption de ces thèmes, en tout cas pas avec une telle force et avec une telle constance. Bien sûr, ils n'apparaissent pas d'entrée dans les témoignages. Ce qui est d'abord massivement décrit comme alimentant les pratiques de déconnexion, c'est (pour reprendre le vocabulaire employé par nos interviewés) la volonté de « souffler», de « prendre du recul», de « faire le vide », de « se mettre à l'écart du tumulte » et même "d'arrêter d'être sonné ». Ce qui est recherché s'inscrit alors dans la même logique de distanciation, de mise à l'écart provisoire, de repos et de silence que l'on peut observer sur le plan professionnel.

Lorsqu'elle n'est pas commandée par ces attitudes de défense ou de recul face à trop d'interpellations ou de sollicitations non désirées, la déconnexion est toujours présentée comme le fruit d'un choix. La déconnexion est alors toujours volontaire et proactive. La déconnexion est dans ce cas souvent expliquée par la défense d'un temps à soi dans un contexte de mise en synchronie généralisée, par la préservation de ses propres rythmes dans un monde poussant à l'accélération, par le droit de ne pas être dérangé dans un environnent télécommunicationnel intrusif, et à la volonté d'être tout à ce que l'on fait dans un entourage portant au zapping et à la dispersion. L'attente, l'isolement et le silence, longtemps combattus, car synonymes de pauvreté, d'enfermement ou de solitude réapparaissent dans ce cadre non plus comme quelque chose de subi mais de choisi.

\begin{abstract}
Ainsi cette enseignante (Julie, 36 ans) qui, plusieurs fois par semaine, fait du jardinage et « ne veut sous aucun prétexte être interrompue par un coup de fil »: «Ce sont des moments de calme où je ne pense à rien. J'ai besoin de ça. Je suis avec mes plantes, je prends soin d'elles, ça me calme » ou ce pharmacien (Léon, 57 ans) qui, dès qu'il le peut, fait de longues marches "surtout sans téléphone » ou encore cet autre enseignant (Philippe, 46 ans) qui «s'applique à prendre tous les jours [son] café sans [son] portable [qu'il fait] exprès de laisser dans [son] bureau ». "Je suis comme Philippe Delerm avec sa première gorgée de bière : je savoure. Je suis là, avec mon café, sans mails, sans SMS, sans rien mais pleinement là et ça me suffit ».

Il s'agit aussi d'être « tout à son interlocuteur », ou « entièrement présent » au lieu où l'on se trouve, ou encore "totalement attentif» à l'événement auquel on assiste, et la déconnexion charge à son tour l'instant, le lieu ou la communication d'une survaleur inédite : celle de l'attention et du partage.

Le fait que tout le monde sorte son portable pour le déconnecter au concert ou au théâtre « magnifie la scène » et crée une sorte « de communauté d'attention ", " on est embarqué dans le même bateau, on est tous là pour la même chose, il n'y a plus rien d'autre » et peut s'avérer très pédagogique : « Eh oui, se déconnecter est possible ! » Il en va de même dans certaines conversations ou réunions où le fait de se déconnecter signifie précisément à ses interlocuteurs que rien n'est plus important ${ }^{26}$. Selon la même logique, ne pas répondre à un appel téléphonique en pleine conversation en face à face relève aussi de ce type de déconnexion attentive.
\end{abstract}

\footnotetext{
${ }^{26}$ Il faut croire que le conseil des ministres ne l'était pas tant que cela pour certains d'entre eux : François Hollande s'est vu contraint (avril 2014) d'y d'interdire les portables...
} 
Ces déconnexions peuvent être très éphémères et sont la plupart du temps partielles et situées (choisir de ne pas relever ses e-mails mais laisser son téléphone ouvert, mettre sur silencieux mais regarder de temps en temps qui a appelé, enclencher son service de géolocalisation pour trouver une adresse mais l'enlever aussitôt après). Il semble que nos contemporains fassent en la matière preuve d'un savoir-faire de plus en plus original au fur et à mesure où les TIC se complexifient. Chacun puise dans son expérience quotidienne pour instaurer des formes de déconnexion adaptées aux situations rencontrées. Toutes relèvent d'une économie de l'attention et mobilisent à la fois un ensemble de règles quasi techniques (connaissance des potentialités de son smartphone et de ses applications), un art de l'évaluation (en termes stratégique ou de bienséance) et une capacité d'action (choix). Il s'agit d'articuler différents types d'engagements sous la forme soit de successions (connexiondéconnexion) soit de modulation (déconnexion e-mails mais pas téléphone, ou déconnexion totale sauf trois numéros entrants, ou filtre visuel, etc.), tout devenant une question de choix et de priorité.

Lorsque la déconnexion n'est pas vécue comme un simple mouvement de repli face à trop d'interpellations, comme une pause ou un instant de repos, et lorsqu'elle n'est pas non plus principalement motivée par la volonté d'être «tout à ce que l'on fait» ou " tout à son interlocuteur» ou encore «tout au spectacle auquel on assiste», alors elle est décrite comme une « coupure », une " prise de distance », une " retraite », visant à « se retrouver », à "faire le point». Elle ouvre un moment ou une période de dialogue de soi à soi, de réflexivité, de confrontation avec le sens de sa vie et de retrouvailles avec son intériorité.

Cette expérience de l'intériorité n'est jamais simple. Elle se pose en tension avec les logiques de reconnaissance et de gain qui motivent la connexion. Lors des déconnexions de ce type, il n'y a en effet plus d'e-mails, plus d'appels ou plus de réseaux sociaux pour attester de son existence aux yeux des autres, plus de tweets ou d'Internet pour informer de la marche de ce monde ! Il n'y a plus de stimulations extérieures, plus de notifications, plus de distractions et d'occupations immédiates. Il n'y a plus rien en dehors des seules empreintes que tout cela a laissé sur soi et qu'il s'agit justement d'ordonner afin de leur donner du sens. Le choix, de l'aveu même de nos interviewés, est toujours délicat et difficile à faire. Il s'agit en effet et en particulier de renoncer, même si ce n'est que pour cinq minutes, à tout ce qui, potentiellement, pourrait parvenir à eux, précisément par ces canaux qu'ils décident de momentanément couper.

Ce à quoi il leur est difficile de renoncer est exactement la même chose que ce qui les pousse à interroger de façon frénétique leur boîte e-mail, leur répondeur ou leurs réseaux sociaux. À cet endroit, beaucoup évoquent des conduites d'addiction et le vocabulaire même employé par nos interviewés pour s'y référer («je suis accro», «c'est une véritable dépendance », " je ne peux pas m'en passer ») peut porter à le penser. Mais il y a dans la plupart des cas contresens en la matière et la médiatisation facile du mot addiction y est sans doute pour beaucoup ${ }^{27}$. Il s'agit bien plutôt de curiosité et d'un énorme désir d'advenance, c'est à dire de quelque chose qui survient et qui donne l'impression à la fois de surprise et de renouvellement constant. Il y a comme une attente diffuse mais constante de se laisser surprendre par de l'inédit et de l'imprévu, par un appel ou un SMS qui va changer le cours de sa journée ou de sa soirée en la densifiant ou en la diversifiant, et en rendant, finalement, sa

\footnotetext{
${ }^{27}$ Les phénomènes d'addiction sont tous liés à de la souffrance. Souffrance qui, lorsque le produit ou l'objet par lequel d'addict est lié sous forme d'une étroite dépendance vient à manquer, indique de graves pathologies. Ce travers, qui consiste à qualifier d'addiction toute pratique régulière (et parfois exagérée) d'une activité, a récemment été dénoncée par l'Académie des Sciences (2013) à l'occasion de la remise d'un rapport sur l'Enfance et les écrans, et dans lequel la distinction entre "pratiques régulières » et addiction est clairement rappelée.
} 
vie plus intéressante et plus intense. De la même façon que, sur Internet, chacun fait sa moisson de nouveautés, certains faisant du hasard leur méthode de recherche en espérant qu'elle se traduise en sérendipité dans le meilleur des cas et en perte de temps dans le pire (le zapping permettant d'exclure les cas malheureux donc, dans cette perspective, l'ennui). Ce n'est donc pas un phénomène d'addiction qui rend la déconnexion difficile, mais bien plutôt la peur de rater quelque chose. Les Américains ont créé un acronyme pour désigner cette crainte : FOMO (Fear Of Missing Out). On la retrouve lorsqu'il s'agit d'introduire un peu de déconnexion sur son poste de travail.

«Couper le son et les pastilles [notifications de nouveaux e-mails reçus] n'y a rien fait. Parce que je continuais à les interroger toutes les 5 minutes (...). Ça mettait du bazar dans ce que j'étais en train de faire, mais je ne pouvais pas [m'empêcher de lire les mails]... Il fallait que j'aille voir, c'était comme un réflexe. » (Christian, 33 ans, comptable)

«Quand je suis connecté, c'est pas loin de l'addiction. D’une certaine manière, la déconnexion de quelques jours, c'est une cure pour moi, un sevrage, une désintoxication totale, comme pour la cigarette. C'est une lutte contre 1'addiction. » (Gérard, 57 ans, formateur)

Lors de sa recherche, Thierry Venin (2013) a posé la question: «vous arrive-t-il, en l'absence de notification, d'appuyer sur le bouton « relever » afin de vérifier si vous n'avez pas, par hasard, manqué un nouvel e-mail ? » et s'est aperçu que pratiquement tous les participants à ses focus-groups (12 de 10 personnes environ) ont répondu oui ! Personne n'y était obligé, mais tout le monde l'avait fait. Venin rapporte comment l'un d'entre eux, étant exceptionnellement resté plus de 20 minutes sans recevoir d'email, en était même venu à téléphoner à son service informatique afin de savoir si le réseau marchait bien...

Mais la même attente est présente au niveau privé, quelle que soit la situation.

« Le premier soir, je n'ai pas pu faire autrement: j'ai rallumé mon portable pour voir si j'avais des SMS et pour dire à [ma femme] que tout était OK. Mais le lendemain, j'ai été voir mes mails. Plusieurs fois de suite. Ça me manquait, c'était plus fort que moi. Ça m'empêchait de faire le point, de prier, mais j'en avais besoin » [ce n'est qu'au bout de trois jours qu'il est parvenu à ne plus interroger ses e-mails et à ne regarder « que deux fois par jour» s'il avait des SMS]. (Claude, 46 ans, banquier, une semaine de retraite dans un monastère).

« Il y a très peu de chance pour qu'on m'appelle pour me dire que je viens d'hériter d'un lointain cousin. C'est pas pour ça que je regarde continuellement mes mails et messages, faut pas rêver... Mais pourtant je le fais et j'attends toujours (...) quelque chose de bien, oui... quelque chose de bien ou de sympa... une bonne nouvelle... Je regarde continuellement, c'est un réflexe, surtout dans les temps morts » (Elise 36 ans, éducatrice spécialisée)

La déconnexion implique toujours une prise de risque. Le fait même de l'assumer est vécu comme une victoire. Victoire sur la crainte de manquer quelque chose, victoire sur la peur de s'ennuyer, victoire de ne pouvoir compter que sur soi.

Cela peut être très modeste, comme Catherine (employée, 52 ans) qui décrit comment le fait d'avoir réussi à aller sans son portable à la pharmacie qui se trouve à 200 mètres de chez elle relève quasiment de l'exploit : « Je me suis dit : je suis capable de le faire ! En fait, il faut être sûr de soi pour s'en passer. On n'est pas sûr que rien ne va pas arriver. Mais bon, on assume (...) Peut-être c'est parce qu'on assume qu'on se sent sûr de soi »; plus prononcé comme Jean (37 ans, instituteur) qui est désormais « capable de rester une demi-journée sans appel et j'assume de rester une demi-journée sans qu'on puisse me joindre. Et je me sens fort! C'est incroyable... une capacité retrouvée à dire non. Non, stop, là... j'ai juste besoin de ne rien faire, de me retrouver ou de réfléchir », ou apparemment beaucoup plus risqué, comme les dizaines de montagnards que nous avons interviewés et qui décident de partir en course sans portable.

Enfin, se déconnecter, c'est aussi prendre le risque de soi-même. Celui de se retrouver seul, vide, sans ressource ou sans but. Ce sont des moments difficiles car (hormis peut-être pour ceux qui croient en Dieu) la confrontation avec le sens de son existence est toujours périlleuse et implacablement angoissante quant à son issue. Ce n'est pas seulement la curiosité ou l'espoir d'advenance qui motive ceux qui, dès qu'ils ont cinq minutes, pianotent 
sur leur clavier. Et ce n'est pas seulement l'ennui qu'ils fuient ainsi : c'est aussi l'angoisse existentielle. Si l'on accepte en effet avec Heiddeger que c'est parce qu'il «s'insère dans le temps » (Zeitigung) que l'individu a conscience de son «être-là » (Dasein), et que cette conscience est avant tout celle de sa finitude individuelle (l'être est dans la conscience du temps qui est celle de sa mort), on peut penser que l'écrasement du présent sur l'immédiat est une façon d'échapper à l'angoisse qu'une telle conscience entraîne. Les TIC sont d'incroyables machines à occuper son temps, à combler tout temps mort en stimulant, informant, instruisant ou distrayant. Mais dans une perspective pascalienne, il est aussi possible de dire qu'elles sont à la mesure de la soif de divertissement de l'homme qui fuit les questions existentielles ou plus simplement celle du sens de la vie.

Se déconnecter c'est donc aussi se mettre à distance du monde. Dans son plaidoyer pour la déconnexion, Rémy Oudghiri (2013) montre combien ce désir de se retirer du monde, prenant selon les cas la forme d'introspection, de méditation, de contemplation ou de prière, est universel et comment il a traversé les siècles. Pour lui, la déconnexion aux TIC est aussi une forme de retraite intérieure, celle-ci étant «le plus sûr moyen de ne pas se laisser engloutir par le flot incessant des vanités de la vie contemporaine » et « le lieu de recherche du sens de sa vie ». C'est une position que nous avons certes retrouvée chez certains de nos interviewés. Par exemple Claude (46 ans, banquier, ayant passé une semaine de retraite dans un monastère) :

«La première chose que j'ai faite en arrivant ici (en fait avant), ça a été d'éteindre mon portable en laissant un message : en cas d'urgence absolue, prévenir (...). J'avais fait la même chose avec mon adresse mail. D'un seul coup, plus rien, ni mails, ni téléphone, ni Internet, rien... Je me suis retrouvé moi seul. À la recherche de Dieu, mais moi seul » (.

Mais c'est moins de cette introspection fondamentale liée à la condition humaine dont nous ont majoritairement parlé ceux qui ont vécu une expérience de déconnexion de quelques jours que d'une réflexivité peut-être plus banale mais beaucoup plus pressante. Celle dictée par l'impératif intérieur de «faire un peu d'ordre» dans leur vie, d'y « voir un peu plus clair » ou de « faire le point ».

« J'ai voulu reprendre en main une existence qui m’échappait. Je m'agitais, beaucoup même... Mais j'étais mécontent (...). Et plus je ressentais [ce mécontentement] et plus je m'agitais. Oui, j'étais hyperconnecté ! Je n'avais pas une minute à moi. J'avais ce qu'on appelle une vie bien pleine. Mais j'étais vide (...). » (Jean, 52 ans, directeur d'un corps consulaire, faisant le chemin de Saint-Jacques de Compostelle)

«Le soir, lorsque je passais tant de temps sur Facebook et après à errer sur Internet, je savais très bien que je cherchais quelque chose autant que je fuyais. Et ce que je fuyais, c'était ma vie, ma vraie vie dans laquelle ça n'allait pas. » (Pierre, 30 ans, ingénieur)

«Quand je suis connecté, je n'ai pas de relation avec moi-même. Mon esprit est toujours sur quelque chose d'autre, dans la distraction. Alors là, j'ai décidé de réfléchir à ma vie, et j'ai vraiment du temps. » (Martin, 34 ans, programmateur)

«Pour tout ce qui est connexion, c'est une fuite, on n'affronte pas la réalité et au lieu de penser à ses problèmes, on est devant son écran et le temps passe, le temps passe (...) et on ne réfléchit pas. Et quand on se déconnecte oui, on réfléchit. On prend des décisions. On est capable de prendre des décisions. Ouais ! » (Patricia, 49 ans, assistance service de promotion en tourisme)

« J'ai été assaillie de questions que je connaissais mais ne voulais pas me poser. » «D'un seul coup tu as le temps de te poser des questions essentielles et tu te dis : mais c'est ça qui est important !» «Là, y'a pas de fuite possible, de trucs pour penser à autre chose : il y a toi et toi. » "Une fois le bruit éliminé, qu'est-ce qui reste comme son ? Quelle est la mélodie de la vie ?»

La déconnexion peut alors se révéler saisissante : à partir du moment où l'état de connexion quasi permanent devient la normalité du quotidien, la déconnexion (si celle-ci se prolonge au-delà de quelques heures et encore plus de quelques jours) représente une telle rupture qu'elle fait presque automatiquement émerger des questions de fond. C'est comme si 
la déconnexion produisait un brusque appel d'air emportant toute agitation, distraction et dispersion pour laisser l'individu seul face à lui-même. Le contraste est tel que l'ennui n'y a souvent plus sa place : la confrontation brutale avec le sens de sa vie occupe tout l'espace. Alors le silence interpelle, la distance questionne et le passé resurgit. La recherche d'une cohérence et d'une continuité de soi, le travail incessant d'une signifiance intime et la défense d'une autonomie se posent alors dans toute leur acuité.

\section{Conclusion : la déconnexion comme épreuve hypermoderne}

Pris dans un courant d'hyper-sollicitations, un flux continu d'informations, emporté par d'incroyables possibilités en termes de connaissance et de distractions, chacun se trouve plongé dans le mainstream véhiculé par les TIC et en vient à être, comme le décrivait David Riesman (1964) il y a plus de 60 ans de cela, extra-déterminé. Il observait alors comment la classe moyenne américaine était en train de passer (c'était en tout cas sa thèse) d'une société d'acculturation normative produisant des individus intro-déterminés (c'est-à-dire ayant intégré des normes sociales de comportement, des principes moraux et un socle éthique leur permettant d'avoir des convictions personnelles) à une société que Zigmun Bauman (2004) aura entre-temps qualifiée de " liquide » où les individus sont extro-déterminés (c'est-à-dire ayant tendance à adapter de façon tactique leurs comportements aux situations rencontrées et au seul regard des autres). Jamais nous n'avons été aussi liés, immédiatement, à autant de personnes, aussi informées d'autant de choses différentes, et aussi susceptibles d'y réagir où que l'on soit. Reprenant la distinction de Riesman, on pourrait dire que la déconnexion tend à réintroduire de l'intro-détermination dans nos vies là où l'extro-détermination ne suffit plus.

Seulement, contrairement au type de société que quittait la classe moyenne américaine des années 1950, la nôtre n'a plus beaucoup de repères à offrir en dehors de l'injonction paradoxale à être authentique et à réussir sa vie. Alors que, jusqu'au seuil des années 1970, un futur chargé d'espoir pouvait encore collectivement être entrevu à travers les idéologies d'émancipations, l'actuelle désaffection envers les utopies laisse place à un présent qu'il s'agit de conquérir personnellement à partir de ses propres ressources. On n'espère plus collectivement dans le futur : il faut réussir personnellement dans le présent. L'individu est mis en face de ses propres réalisations. Démiurge de lui-même, il n'est plus l'objet d'un destin, mais semble être le seul maître de sa vie. Alors que, jusqu'à peu, les normes sociales disciplinaient l'individu et le portaient à accepter sa condition (produisant conformité ou, au contraire, révolte), elles l'assignent aujourd'hui à devenir " lui-même » sans autre référence que sa propre volonté. C'est en cela que la déconnexion est une épreuve pour l'individu contemporain : si son environnement social et, précisément, ses connexions l'assignent à chercher sa voie et à en assumer la direction, la déconnexion le confronte à l'impératif de leur donner un sens.

Ce que la modernité offrait déjà il y a un siècle (la capacité instrumentale à agir rationnellement sur le réel) se trouve décuplé dans notre rapport au monde, en particulier grâce aux TIC, et cet emballement occupationnel ne cesse d'être magnifié. C'est essentiellement sous cet aspect que le concept d'hypermodernité a été pensé, c'est à dire comme l'approfondissement, la radicalisation et l'extension de ce sur quoi la modernité s'est fondée : la raison, le mouvement, le choix, l'inédit et l'efficience. Même si c'est souvent pour en dénoncer les extrêmes ou les excès, les auteurs recourant au concept d'hypermodernité pour rendre compte des traits les plus novateurs des sociétés contemporaines insistent tous sur cette dimension de radicalisation de l'action instrumentale mue par un désir d'efficience ${ }^{28}$. Mais l'hypermodernité ne se résume pas à cela. Si l'on accepte en effet que la modernité est bien ce moment historique où le décalage de soi à soi est socialement reconnu et quasi

\footnotetext{
${ }^{28}$ En français, voir l'ouvrage collectif L'individu hypermoderne (dir. Nicole Aubert, 2004).
} 
institué, c'est-à-dire où s'ouvre, pour l'individu, ce fossé qui ne sera jamais plus comblé entre son désir d'être et ce qu'il est ou, dit autrement, entre sa subjectivité et son existence sociale, alors on peut aussi définir l'hypermodernité comme l'approfondissement de ce fossé et comme l'extension de cet écart, de ce décalage de soi à soi à des couches de plus en plus nombreuses de la population et sous une forme de plus en plus pressante. L'autre pan de la modernité, la réflexivité, l'intériorité, la subjectivité individuelle connait le même approfondissement et la même extension que le premier. La différence réside en ce que l'un se fait au grand jour, l'autre en silence, que l'un est facile, aisé et immédiat tandis que l'autre demande un effort, nécessite du temps et pose plus de questions qu'il n'en résout.

La mise à distance du monde, qui a accompagné son objectivation et sa rationalisation, a permis de le rendre plus maîtrisable et contrôlable. Comme le souligne Giddens (1994), le développement technologique et organisationnel a délivré l'individu des dangers et craintes situationnelles d'autrefois. Mais l'approfondissement du caractère objectif de la vie, en désamorçant les réponses sociales «enchantées » qu'apportaient mythes et traditions au questionnement existentiel, est aussi celui de l'insécurité ontologique. Celle-ci conduit l'individu à un nécessaire travail de construction de son existence de telle sorte qu'elle puisse avoir un sens à ses propres yeux. En somme, en l'absence d'un traitement social, l'individu doit trouver en lui-même le sens de son existence et surtout en expérimenter l'efficience sous la forme d'un contentement et d'un sentiment de vérité. La radicalisation instrumentale du réel se traduit chez l'individu par un besoin de préserver sa propre subjectivité sous une forme d'authenticité de soi à soi et de soi aux autres. C'est à cette préservation que renvoie en partie la déconnexion et c'est parce qu'il ne s'agit pas là de quelque chose de simple à assumer que l'on peut en parler en terme d'épreuve.

Il n'y a pas de raison d'opposer mécaniquement connexion à déconnexion : il semble que l'une n'aille pas sans l'autre. Ou plus exactement la connexion maîtrisée (où en tout cas sa recherche) implique des formes de déconnexion, de la même façon que la déconnexion n'a de sens que parce que, le reste du temps, il y a connexion. Les extrêmes se réunissant, une connexion non maîtrisée peut conduire à une dissolution de soi dans une hyperconnexion déstructurante ou à une déconnexion implosive (burn out). C'est pour cela que l'hypothèse d'une postmodernité n'est pas à exclure : la distraction et l'effervescence dans une relation consumériste au réel et aux autres recouvrent alors l'écart réflexif moderne et le soi se disloque dans de multiples identifications éphémères au gré des rencontres et de zapping dans des réseaux de plus en plus aléatoires. Rapportée à la consommation médiatique, ce laisseraller post-moderne pourrait, comme le remarque Edgar Morin (1981, p. 26) s'avérer dangereux: "l'excès d'information étouffe l'information quand nous sommes soumis au déferlement ininterrompu d'événements sur lesquels on ne peut méditer parce qu'ils sont aussitôt chassés par d'autres événements (...) Alors que l'information apporte forme aux choses, la surinformation nous plonge dans l'informe. » Pour pouvoir " donner forme » aux informations, une prise de distance est nécessaire. Pour cesser d'être étourdi par leur défilement, troublé par leurs couleurs changeantes et ébloui par l'éclat de leur nouveauté, il faut savoir prendre du recul, être capable d'arrêter leur déferlement. À cette seule condition ces mêmes informations peuvent être rapportées à un système de représentations et de significations qui permet, par la comparaison, la confrontation et la vérification, de formuler une interprétation ${ }^{29}$.

La Boétie décrivait la servitude volontaire comme la part de ce que l'on délègue de notre liberté pour pouvoir vivre dans un monde praticable par le plus grand nombre. Ici la

\footnotetext{
${ }^{29}$ Sur ce sujet, l'analyse de Gérard Claisse (1997, pp. 123-124) est bien pessimiste : « À l'interconnexion en temps réel de nos prothèses informationnelles, répond la déconnexion généralisée de nos capacités à comprendre, à mettre en perspective, à contextualiser, à interpréter les informations qui nous parviennent. L'information entre, sort, circule, mais ne s'arrête pas. La prolifération d'informations nous rend aveugles. »
} 
déconnexion volontaire renvoie à ce que l'on accepte de renoncer en sécurité, en informations ou en distractions pour pouvoir préserver un quant-à-soi, un espace privé, un anonymat, une distance réflexive dans lesquels l'individu peut se penser comme sujet. Si, rapporté aux TIC, l'acteur est celui qui communique dans les réseaux, le sujet est celui qui donne un sens à cette communication. Mais pour cela, il doit être capable de se déconnecter de temps en temps. Et c'est en cela que le va-et-vient entre connexion et déconnexion est un parfait indicateur de l'hypermodernité et de l'incertitude qu'elle ne cesse de produire.

\section{Bibliographie}

Académie des Sciences (2013), L'enfant et les écrans, Paris, Institut de France

Aubert N. (2003), Le culte de l'urgence. La société malade du temps, Paris, Flammarion.

Aubert N. (dir., 2004), L'individu hypermoderne, Toulouse, Erès.

Bauman Zygmunt (2004), L'amour liquide. De la fragilité des liens entre les hommes, Rodez, Le Rouergue/Chambon.

Bonneville L. et Grosjean S. (2012), « Pourquoi faut-il sortir de la parole instrumentalisée en milieu de travail ? », Communication, vol. 30, $\mathrm{n}^{\circ} 2$, pp. 1-21.

Brotcorne P. et Valenduc G. (2009), «Les compétences numériques et les inégalités dans les usages d'Internet », in Les cahiers du numérique, vol. 5, n 1, pp. 45-68.

Claisse G. (1997), L’abbaye des télémythes, Lyon, Aléas.

Crouzet T. (2011), J'ai débranché, Paris, Fayard.

Dubet F. (1994 ), Sociologie de l'expérience, Paris, Seuil.

Ehrenberg A. (1995), L’individu incertain, Paris, Calmann-Lévy.

Felio C. (2013), Pratiques communicationnelles des cadres. Usage intensif des TIC et enjeux psychosociaux, thèse de doctorat, Université de Bordeaux 3.

Hargittai E. (2002), « Second-Level Digital Divide : Differences in People's Online Skills », First Monday, vol. 7, $\mathrm{n}^{\circ} 4$.

http://firstmonday.org/htbin/cgiwrap/bin/ojs/index.php/fm/article/view/942/864

Heidderger M. (1986) [1927] : Etre et temps, Paris, Gallimard.

Jauréguiberry F., «L'usage du téléphone portatif comme expérience sociale », Réseaux $\mathrm{n}^{\circ} 82$ 83, 1997, pp. 149-164.

Jauréguiberry F. (2003), « Les branchés du portable », Paris, PUF.

Jauréguiberry F. (2005), "L’immédiateté télécommunicationnelle », in Nouvelles technologies et mode de vie (éd. Moati Ph.), La Tour d'Aigues, l'Aube, 2005, pp. 85-98.

Kaufmann J.-C., (2004), L'invention de soi, Paris, Armand Colin

Lahire B. (1998), L'homme pluriel, Paris, Nathan

Martuccelli D. (2010), La société singulariste, Paris Armand Colin

Maushart S. (2013), Pause, Paris, Nil.

Oudghiri R., (2013), Déconnectez-vous !, Paris, Arléa. 
Ricoeur P. (1990), Soi-même comme un autre, Paris, Seuil

Riesman D. (1964) [1952], La foule solitaire, Paris, Arthaud.

Rogers E. (1983), Diffusion of innovations, New York, Free Press.

Simmel G. (2013) [1903], Les grandes villes et la vie de l'esprit, Paris, Payot.

Turkle S. (2011), Alone Together. Why we expect more from Technology and less from each other, New York, Basic Books.

Van Dijk J. (2005), The Deepening Divide. Inequality in the Information Society, Thousand Oaks, Sage.

Venin T. (2013), Techniques de l'Information et de la Communication et risques psychosociaux sur le poste de travail tertiaire, thèse de doctorat, Université de Pau.

\section{Résumé}

Les conduites de déconnexion apparaissent comme une volonté de maîtrise des technologies de communication. Elles sont toujours ponctuelles et la plupart du temps partielles. Il s'agit de ne pas se laisser envahir par trop d'informations non désirées, d'échapper à un mode d'interpellations incessantes, à l'urgence et à la pression managériale, au contrôle hiérarchique ou à l'impression d'être surveillé. La déconnexion équivaut alors à reprendre souffle et distance, à retrouver son rythme et un temps à soi. Mais elle est aussi parfaitement révélatrice de la figure de l'homme hypermoderne qui ne se contente pas de suivre l'accélération du mouvement moderne (capacité instrumentale à agir rationnellement sur le réel et soif d'inédit et de changement) mais l'interroge au contraire par une réflexivité accrue sur ses choix et dans l'inquiétude qui en résulte. 